\title{
Interplay of Electroencephalogram Phase and Auditory-Evoked Neural Activity
}

\author{
Stepan Y. Kruglikov ${ }^{1,2}$ and Steven J. Schiff ${ }^{1,3}$ \\ ${ }^{1}$ Krasnow Institute for Advanced Study, ${ }^{2}$ School of Computational Sciences, and ${ }^{3}$ Department of Psychology, George Mason University, Fairfax, Virginia 22030
}

\begin{abstract}
Auditory-evoked potentials (AEPs) were triggered in real time as a function of ongoing electroencephalogram (EEG) phase. Phase triggering on-line or retrospective phase-selective averaging introduces phase artifacts such as spurious troughs or peaks, which mask mid-latency and affect the amplitude of late AEPs. We developed a method to control for phase artifacts by phase-selective averaging of trials, recorded without stimulation, and used this to uncover a previously unknown phase dependency of AEPs. Not only are such findings inconsistent with the standard additive evoked potential model, but we identified clear neural correlates at fixed latencies, which are inconsistent with the recently proposed phase-resetting model. Our findings suggest that a new conceptualization is required to account for the interplay between the correlates of neural-evoked activity and modulation of ongoing EEG that together constitute evoked potentials.
\end{abstract}

Key words: evoked; potential; phase; neural correlate; real time; mid-latency

\section{Introduction}

Since the earliest electroencephalogram (EEG) recordings, it has been suggested that the response of the brain to sensory stimuli might be dependent on spontaneous oscillatory activity of the cortex. In 1933, Bishop suggested that there was a spontaneous rhythmic variation in cortical excitability and that the timing of stimuli within this cycle determined the responsiveness of the cortex (Bishop, 1933). By following the suggestion that cortical responses fluctuated with intrinsic alpha $(8-13 \mathrm{~Hz})$ rhythms (Lindsley, 1952), others have shown that visual (Callaway and Yeager, 1960; Dustman and Beck, 1965) and auditory (Rice and Hagstrom, 1989) stimulated behavioral response latencies were dependent on the phase of the alpha rhythm and that the amplitude of electrical evoked potentials was similarly dependent (Jansen and Brandt, 1991). Concurrent with mounting evidence that ongoing cortical activity influences sensory processing (Rahn and Başar, 1993; Arieli et al., 1996; Polich, 1997; Kisley and Gerstein, 1999; Tsodyks et al., 1999), the hypothesis that sensory evoked potentials (EPs) are a manifestation of phase resetting of EEG has been proposed (Brandt, 1997; Tass, 1999; Makeig et al., 2002; Jansen et al., 2003). If EPs are dependent on ongoing EEG phase, then the customary hypothesis (Lopes da Silva, 1999) that the responses to randomly timed stimuli are equivalent for averaging is incorrect (Tass, 1999; Makeig et al., 2002).

There have been several studies that have used real time triggering of sensory stimuli from the phase of the ongoing alpha rhythm (Callaway and Yeager, 1960; Bechtereva and Zontov, 1962; Dustman and Beck, 1965; Remond and Lesèvre, 1967).

\footnotetext{
Received June 9, 2003; revised Sept. 16, 2003; accepted Sept. 19, 2003.

This work was supported by National Institutes of Health Grants F31DC05651 (to S.K.) and K02MH01493 (to S.S). We are grateful to J. Polich for helpful comments on this manuscript and for the technical expertise and discussions with Bruce Gluckman, Paul So, Ben Munyan, Paul Rapp, and Steven Weinstein.

Correspondence should be addressed to S. J. Schiff, Krasnow Institute, MS 2A1, George Mason University, Fairfax, VA 22030. E-mail: sschiff@gmu.edu.

Copyright $\odot 2003$ Society for Neuroscience $\quad$ 0270-6474/03/2310122-06\$15.00/0
}

These studies focused on either the late electrical EPs or behavioral responses, but the methodology did not control for the effect of phase artifacts, which manifest themselves as spurious features (troughs or peaks) at stimulus onset and beyond. For instance, if a well defined EEG phase corresponds to a peak or trough in the signal, phase-triggered averaging of an ensemble of such records will reveal a deep trough or peak, followed by a characteristic waveform that is an artifact of the averaging rather than a response to a stimulus.

In all previous work to our knowledge, whether EEG phase was used in real time or analyzed retrospectively, phase was not dissociated from the neuronal correlate of the sensory response. We here introduce controls for EEG on-line phase, using phase triggering with and without applied stimuli. We find that the amplitudes of mid-latency (P30, P50) EPs are strongly dependent on the phase of the EEG, but there are clear neural correlates at these fixed latencies not attributable to phase resetting. We hypothesize that a new conceptualization, beyond the standard (see Fig. 1AI) or phase reset models (see Fig. 1AII), would be required to account for the interplay between the correlates of neural evoked activity and modulation of ongoing EEG that constitute EPs (see Fig. 1AIII).

\section{Materials and Methods}

Experimental procedures. Twenty normal healthy subjects (10 males), 20 -30 years old, were recruited from the campus of George Mason University under a protocol approved by the university's Institutional Review Board. Subjects took no prescription medications and had no hearing problems. They were paid \$50 US after testing.

EEG/EP equipment was modified to present auditory stimuli timed to the phase of the ongoing EEG (Fig. $1 B$ ). Using phase in real time is complicated by the fact that the methods generally used to calculate phase directly, such as Hilbert or wavelet transforms, are acausal, i.e., require information about the signal in the future from the time point at which the phase is calculated, and cannot be applied in real time (Bendat and Piersol, 2000). Therefore, for each subject the voltages during a 100 
A
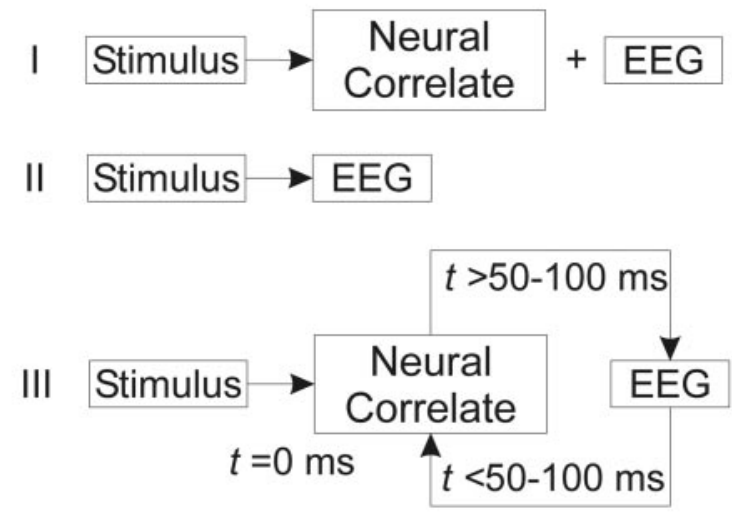

B
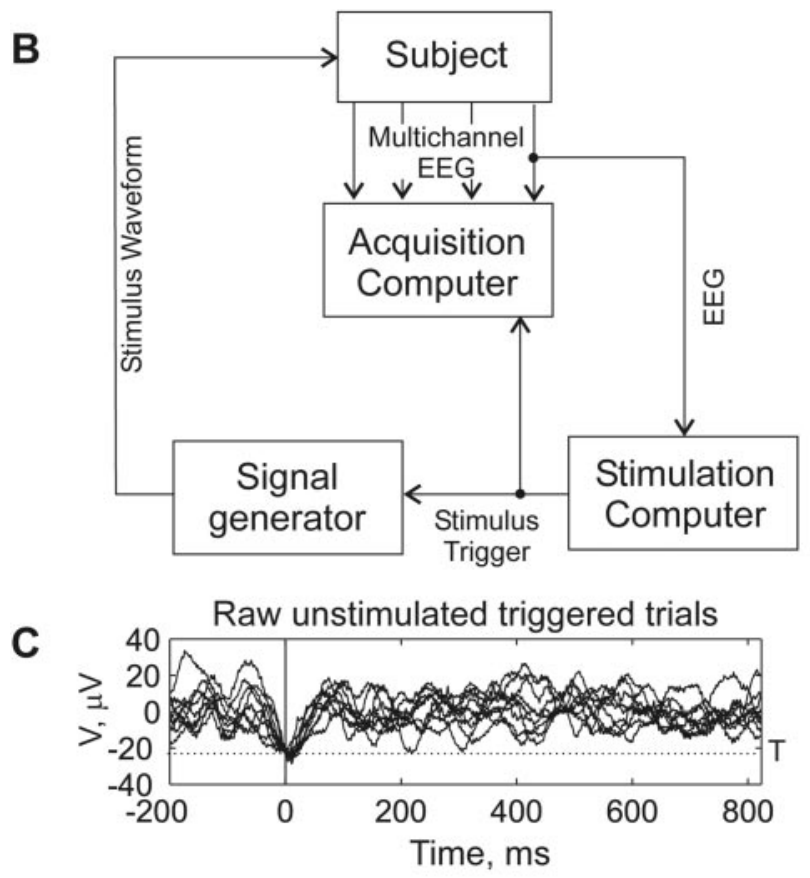

D

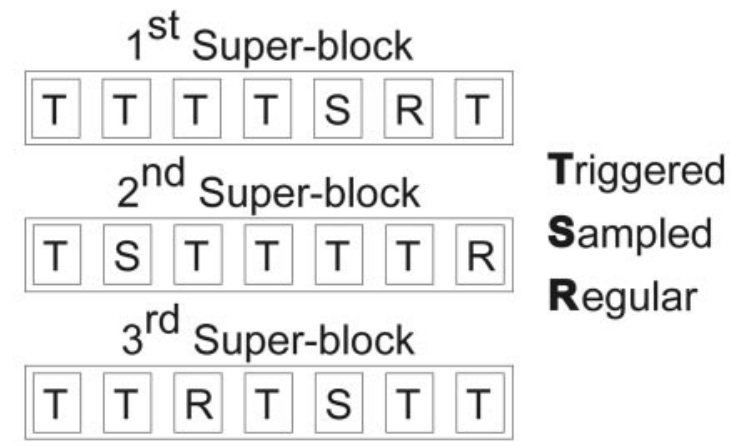

Figure 1. A, Three schemata illustrating the interaction among stimuli, $\mathrm{EEG}$, and the neural correlates of evoked potentials: I, traditional view of stimuli interacting with neural circuitry independently of EEG; II, phase-resetting view of stimuli interacting with EEG; III, schema suggested by the results of this study whereby neural correlates of stimulation and ongoing EEG modulate each other in a time-dependent manner. B, Schematic of experimental apparatus. $C$, Example of 10 raw, unstimulated phase-triggered trials from an individual subject. D, Example of experimental protocol for a subject.

sec baseline recording at the beginning of the experiment (relaxed, eyes closed) were used to set a threshold to identify the most negative $1 \%$ of the amplitudes. Using a threshold that corresponded to deep troughs in the EEG was found to identify signals with nearly identical phases (calculated retrospectively). Ten unstimulated triggered individual trials are shown in Figure $1 C$, visually illustrating how phase is aligned at $0 \mathrm{msec}$. By selecting various delays $(0,25,50,75 \mathrm{msec})$ that followed this threshold, we could trigger stimuli in a phase-dependent manner. In Figure $4 \mathrm{~A}$, we illustrate the statistical significance of such phase dependence.

Four types of trials were presented. The first two types used auditory stimuli that were timed to EEG phase (with four different delays) matched with unstimulated phase control trials in which phase was determined via threshold but in which no stimuli were presented. To match unstimulated phase control trials to phase-triggered stimuli, we shifted control trials backward in time by 25,50 , or $75 \mathrm{msec}$ as required. We thus could control for the waveform morphology associated with EEG phase by subtracting this from the trials with auditory stimuli that were triggered from EEG phase.

This control has not been used previously. Without such a control, phase-selective averaging of EEG creates the "prestimulus phase bias" (Makeig et al., 2002), which has been observed in previous work (Jansen and Brandt, 1991). Use of a phase control allowed us to extract the neural correlates of the EP from the phase-dependent waveform.

For further comparison we presented two types of stimuli without regard to ongoing EEG phase: one set at regular intervals of $3.5 \mathrm{sec}$ with random jitter of $\pm 0.3 \mathrm{sec}$ and another set that used interstimulus intervals resampled from the phase-triggered trials. EEG-dependent triggering increases the variability of interstimulus intervals (Rahn and Başar, 1993), and it is known that longer and irregular intervals can increase the amplitude of EPs (Makeig, 1990). To take this into account, we presented stimuli with intervals sampled from the previous phase-triggered trials, presenting them in blocks without regard to phase.

Because of the clinical interest of paired pulse P50 suppression for the study of sensory gating, we delivered a second auditory stimulus at a fixed 500 msec delay (Freedman et al., 1987). Our working hypothesis was that the response to the second tone also might be affected by the phase at which the first tone was presented.

An example of our block design is shown in Figure $1 D$. Three superblocks containing equal numbers of each type of stimuli were presented. Each super-block contained seven blocks with 50 stimulus presentations in each block. The order of the blocks was randomized within each superblock, with the restriction that the sampled stimulus interval block could not occur before at least two phase-triggered blocks were performed. Fifteen random sequences of 50 phase-triggered trials were created, each sequence consisting of 10 presentations of each of the five types of phasetriggered stimuli. Five of these sequences were presented in random order within each super-block so that in total 750 phase-triggered stimuli were given (150 of each type) in the entire experiment. To ensure uniformity, we used the same 15 random sequences (with the same stimulus type order) for each subject but in randomized block orders. The sampled interval blocks were constructed by randomly selecting phasetriggered intervals from the pool of all previously triggered intervals $(0$, $25,50,75 \mathrm{msec}$ and unstimulated phase control trials).

Subjects were relaxed with eyes closed during stimuli and were asked to open their eyes and stay alert between blocks. Each block lasted from 3 to $5 \mathrm{~min}$, and the entire experimental protocol required on average 90 min to complete. In all, 17 of 20 subjects completed the entire protocol (150 trials of each type), and the remaining three ( 3 of 20) subjects completed two of three super-blocks (100 trials of each type).

Stimuli were produced by a signal generator, which generated two 20 msec $1000 \mathrm{~Hz}$ tones, $500 \mathrm{msec}$ apart, at $65 \mathrm{~dB}$ sound pressure level above hearing threshold at $1000 \mathrm{~Hz}$, delivered to the subject via insert earphones (Etymotic Research model 3A, Elk Grove Village, IL). An EEG cap (Neuroscan QuickCap, El Paso, TX) with Ag-AgCl electrodes was applied according to the 10-20 system, and EEG passed through a biopotential isolation unit (Grass IMEB-2NUM25, Quincy, MA), was analog-filtered $(0.3-100 \mathrm{~Hz},-3 \mathrm{~dB}$ ), amplified with gain 10,000 (Grass model 12), digitized at $1 \mathrm{kHz}$ across 12 bits (Digidata 1200A, Axon Instruments, Union City, CA), and recorded on an acquisition computer The width and roll-off of the analog bandpass filter did not distort phase significantly nor create appreciable phase delay in the region of the dominant EEG frequencies in the alpha band. No further on-line digital filtering was applied before determination of threshold or retrospective analysis of phase. Electrodes were applied with conductive gel, and im- 
pedances were kept below $5 \mathrm{k} \Omega$, using electrodes F3, Fz, F4, C3, Cz, C4, P3, Pz, P4, bipolar horizontal electrooculogram (HEOG), bipolar vertical electrooculogram (VEOG), and linked ear references. Single-channel EEG from electrode $\mathrm{Cz}$, which yields the largest amplitude mid-latency EP responses (Nagamoto et al., 1991), was digitized simultaneously at $2500 \mathrm{~Hz}$ across 16 bits (PCI-MIO-16XE10, National Instruments, Austin, TX) for a separate stimulation computer, which controlled the signal generator.

Phase was calculated in broad band from Hilbert transformation (HT) of the signal. HT is defined as:

$$
h(t)=\frac{1}{\pi} \lim _{\varepsilon \rightarrow 0}\left\{\int_{-\infty}^{t-\varepsilon} \frac{x(\tau)}{t-\tau} d \tau+\int_{t+\varepsilon}^{+\infty} \frac{x(\tau)}{t-\tau} d \tau\right\},
$$

where $x(t)$ is the original signal (Bendat and Piersol, 2000). The Gabor analytic signal $Z(t)$ is defined as:

$$
Z(t)=x(t)+i h(t)=a(t) e^{i \varphi(t)},
$$

and the phase of the signal can be obtained as:

$$
\varphi(t)=\tan ^{-1} \frac{h(t)}{x(t)} .
$$

Numerically, the Gabor analytic signal was obtained by calculating fast Fourier transform (FFT) of the signal, multiplying the result by:

$$
s(f)=\left\{\begin{array}{c}
2, f>0 \\
1, f=0 \\
0, f<0
\end{array},\right.
$$

where $f$ denotes frequency, and then performing inverse FFT of the product (Bendat and Piersol, 2000). Note that the fourquadrant inverse tangent was used; therefore, the phase was within the interval $[-\pi ; \pi]$.

Phase was determined in broad band $(0.3-100 \mathrm{~Hz})$, and this study was performed without narrow band filtering of signals. On the one hand, the signals of interest, P30 and P50, lay outside, for instance, the alpha (8-13 $\mathrm{Hz}$ ) band often selected for such phase studies. Furthermore, broad band phase assignments via Hilbert transformation are a powerful means to assign phase to biological signals and may avoid possible artifacts introduced during phase assignment on narrow band-filtered signals (Netoff and Schiff, 2002). Although there was prominent alpha band power in many of our traces, the method we present is applicable regardless of the dominant (e.g., alpha, theta, etc.) cortical cycling that is present. Our approach is to apply the least amount of selective filtering of these signals as possible.

Data analysis. Epochs were extracted from 200 msec before until 823 msec after stimulation onset (1024 discrete voltages). Artifact rejection was applied, rejecting epochs if the amplitude in the bipolar HEOG or VEOG exceeded $75 \mu \mathrm{V}$ in absolute value.

Standard procedures were used to identify P30 and P50. P30 peak-topeak amplitude was found as the peak at the maximum between 25 and $45 \mathrm{msec}$ (Kisley et al., 2001) after stimulus onset, and its amplitude was measured with respect to the preceding negativity. P50 was determined similarly in the window of 45-85 msec (Nagamoto et al., 1991; Jin et al., 1997). Before the extraction of P30 and P50 amplitudes we applied a $10-50 \mathrm{~Hz}$ bandpass digital fifth-order Butterworth filter with $3 \mathrm{~dB}$ rolloff, applied with zero phase shift filtering technique (forward and reverse), using MATLAB function "filtfilt" (MathWorks, Natick, MA).
Such a bandpass filter reduces the effect of N100 (Jerger et al., 1992) and commonly is used to extract the positive EP peaks near 30 (P30) and 50 (P50) msec.

The significance of the effect of delay on EP components also was evaluated by performing a bootstrap analysis on the pool of phasetriggered trials from all subjects. This bootstrap tested the null hypothesis that there was no effect of delay on EP amplitude and was constructed as follows. First, of a possible 3000 epochs at each delay we retained 2850 after subject task incompletion (loss of 150 epochs). After artifact rejection $(\sim 10 \%$ rejected) there remained $2640,2663,2668$, and 2649 phasetriggered epochs of each type. For each subject the average of unstimulated phase controls, appropriately shifted to match delays, was subtracted from each phase-triggered stimulated trial. The pool thus consisted of all controlled phase-triggered trials from all subjects at each of the four phase delays. We chose the average, 2655, as the number of trials to form resampled (bootstrap) averages. From the pool of all phasetriggered trials $(n=10620)$ we randomly, without regard to the phase, selected 2655 trials and calculated a bootstrap average. The procedure of resampling and averaging was repeated 1000 times. The 1000 new averages formed a distribution from which the probability of a given voltage at each time point could be determined. We selected confidence limits corresponding to the probability that a given voltage value would have $p \leq 0.025$ chance of exceeding the value. To evaluate individual P30 and P50 peak-to-peak amplitudes, we realigned the preceding negativities before P30 and P50 to zero voltage by subtracting the average value of the preceding negativity (N20 and N40) from each individual epoch, and we recalculated the confidence intervals to determine the significance of 


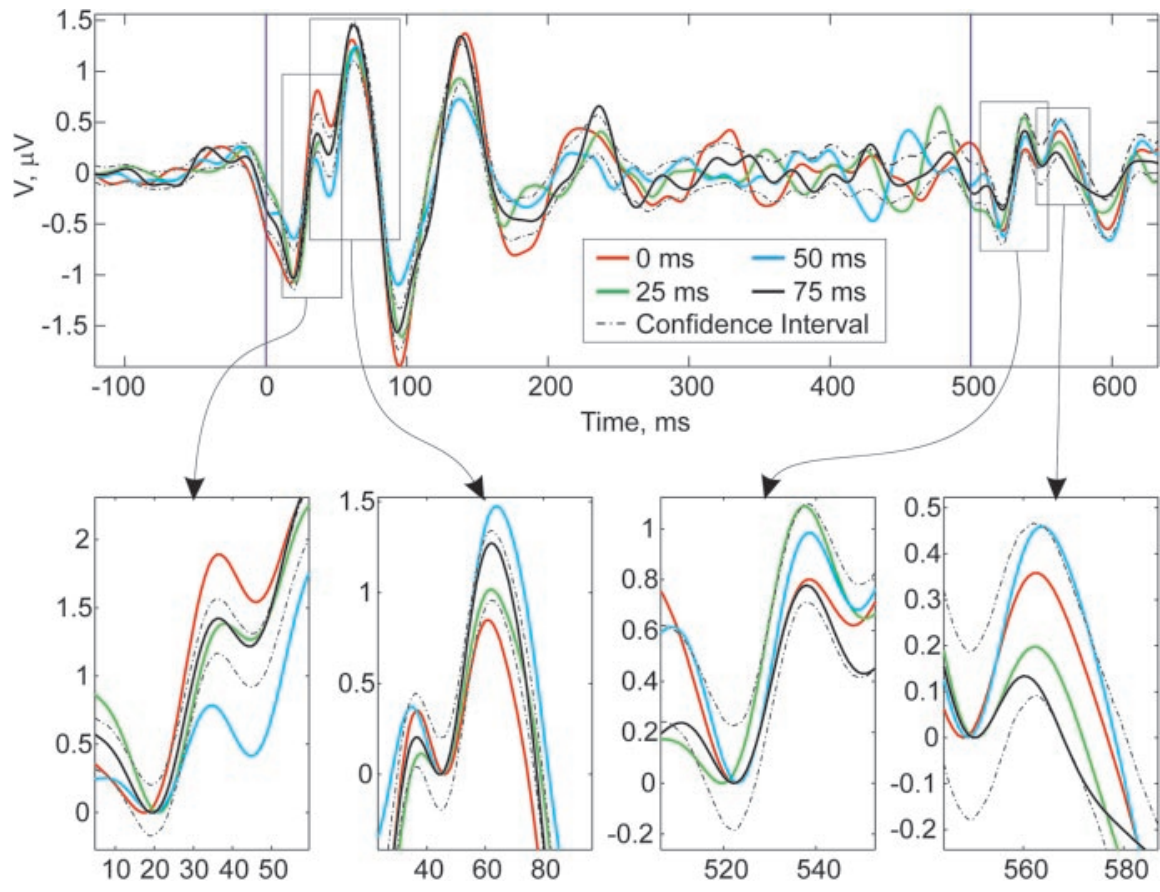

Figure 3. Grand average results from 20 subjects. Solid lines represent averages at 0 msec (red), $25 \mathrm{msec}$ (green), $50 \mathrm{msec}$ (blue), and $75 \mathrm{msec}$ (black). Dashed lines indicate bootstrapped confidence intervals ( $p=0.025$ ). Insets demonstrate increases and decreases in amplitude of P30 at 0 and 50 msec, respectively, and a comparable decrease and increase in P50 amplitude at 0 and $50 \mathrm{msec}$, respectively. Inset waveforms are aligned as detailed in Materials and Methods. No significant effects of first stimulus phase are seen in P30 and P50 measured at the second stimulus 500 msec later.

peak-to-peak excursions. This process was repeated for each EP amplitude shown in the insets of Figure 3.

\section{Results}

An example of the relationship of calculated phase to raw EEG is shown in Figure $2 \mathrm{~A}$. Phase goes through zero at the positive peaks in the EEG signal and abruptly shifts from $\pi$ to $-\pi$ at the troughs. By triggering off the large troughs, we could determine (at $0 \mathrm{msec}$ delay) phase precisely from amplitude. The differences between averages of the phase-triggered and unstimulated control trials are shown in the top panels of Figure $2 B$ for delays of $0,25,50$, and $75 \mathrm{msec}$ after threshold. In the bottom panels of Figure $2 B$ are shown the average responses to regular and sampled irregular stimuli. Note that P30 and P50 average latencies are not affected by these different stimulus conditions (dashed vertical lines in Fig. $2 B$ ). This absence of effect on latency was confirmed for all subjects by repeated measures ANOVA: P30 ( $\mathrm{df}=3, F=1.72$, $p=0.19)$ and P50 $(\mathrm{df}=3, F=1.54, p=0.22)$.

To illustrate the difference between EEG phase with and without stimuli, we plot for one subject the average responses to stimulated and unstimulated phase control trials for $0 \mathrm{msec}$ delay in Figure $2 C$. At the expanded time scale in the middle panels of Figure $2 C$, one sees that the P30 and P50 are attributable to amplitude fluctuations in the signals, with little shift in the phase of the dominant EEG frequency. This is in clear contrast to the apparent phase shift in the trough that accounts for a significant fraction of the N100. Note in the bottom panel of Figure $2 C$ the bandpass-filtered trace $(10-50 \mathrm{~Hz})$ used to extract P30 and P50 amplitudes (Jerger et al., 1992).

Grand average results for all 20 subjects are shown in Figure 3. We resampled epochs without regard to phase to build a bootstrapped confidence statistic that tested the null hypothesis that phase was irrelevant to evoked potential amplitude (see Materials and Methods). As can be seen in the insets of Figure 3 recalculated to evaluate each P30 and P50, the response to the first stimulus was affected strongly by the estimated phase of the EEG, whereas the P30 and P50 amplitudes in response to the stimulus 500 msec later had no such effect. Whereas the first P30 amplitude was increased significantly at 0 msec delay and decreased significantly at $50 \mathrm{msec}$ delay, the amplitude of first P50 was decreased significantly at 0 msec delay and increased significantly at 50 msec delay. At 25 and 75 msec delays no significant effect on peak amplitudes was observed.

The interstimulus intervals (ISIs) for sampled triggered stimulation were significantly longer (mean, $5.1 \mathrm{sec}$; median, 4.4 sec; $\sigma=2.3$ ) than for regular stimulation (mean, $3.5 \mathrm{sec}$; median, $3.5 \mathrm{sec} ; \sigma=0.17$ ) by $t$ test $(p<0.001, t=-36, \mathrm{df}=5388)$. Nevertheless, we observed no significant difference between EP amplitudes for regular and sampled triggered stimulation: P30 $(t=-0.39, p=0.70)$ and P50 $(t=$ $-0.34, p=0.73)$.

We examined the relationship between phase delay and, retrospectively, the actual calculated phases of the EEG at the time of stimulus onset in Figure 4. The relationship between 0 delay and phase was extremely precise to $\pi /-\pi$. As delay was increased, the distribution of phases became spread out and was far more uniform at $50 \mathrm{msec}$ delay than at $0 \mathrm{msec}$ (left and middle columns, Fig. $4 A$ ). We used Rayleigh statistics as a test for randomness against a unimodal distribution (Fisher, 1993) for all phase distributions shown in Figure $4 A$. This statistic sums vectors of the phases ("phasors") to obtain an amplitude, $R$, which varies from 0 (uniform distribution of phases) to 1 (identical) phases. In each panel of Figure $4 \mathrm{~A}$ we show the $R$ values along with Bonferroni-corrected (16 comparisons) significance $\left(p^{\prime}\right)$. The Rayleigh statistics confirm the significant association of phase with delay at 0,25 , and $50 \mathrm{msec}$ for both unstimulated and stimulated trials.

We further tested whether there was any difference between the stimulated and unstimulated distributions at the different delays. Circular rank-based $W$ statistics (Fisher, 1993), which test whether two circular distributions are identical, failed to reveal any differences between phase distributions from trials with stimulation and phase control trials $\left(W_{0 \mathrm{msec}}=0.22, p=0.90 ; W_{25}\right.$ msec $=0.55, p=0.76 ; W_{50 \mathrm{msec}}=0.26, p=0.88 ; W_{75 \mathrm{msec}}=1.06$, $p=0.59)$.

There were very significant (opposite) effects on P30 (0 vs 50 msec delay) and P50 (0 vs $50 \mathrm{msec}$ delay) peak-to-peak amplitudes, shown in the left column of Figure $4 B$, by parametric paired $t$ tests $\left(p<0.001 ; t=8.3\right.$ for $\mathrm{P} 30_{0 \mathrm{msec}} \mathrm{vs} \mathrm{P} 30_{50 \mathrm{msec}} ; t=$ -4.7 for $\left.\mathrm{P}_{50} 0_{0 \mathrm{msec}} \mathrm{vs} \mathrm{P}_{50} 0_{50 \mathrm{msec}}\right)$ and nonparametric Wilcoxon signed rank test $\left(p<0.001 ; z=3.9\right.$ for $\mathrm{P} 30_{0 \mathrm{msec}} \mathrm{vs} \mathrm{P} 30_{50 \mathrm{msec}} ; z=$ -3.5 for $\mathrm{P} 50_{0}$ msec $v s \mathrm{P} 50_{50} \mathrm{msec}$ ); phase had a significant effect by repeated measures ANOVA with delay as a factor $(\mathrm{df}=3,57, F=$ $23, p<0.001$ for P30; $\mathrm{df}=3,57, F=10, p<0.001$ for P50) as well as the Friedman test (Glantz and Glantz, 2001), a nonparametric analog to this ANOVA ( $\mathrm{df}=3, \chi^{2}=31, p<0.001$ for P30; $\mathrm{df}=$ $3, \chi^{2}=17, p<0.001$ for P50). 
For normal subjects the P50 sensory gating ratio, defined as a fraction of the response to the second tone amplitude to the first, should be less than one (Freedman et al., 1987). We found that the P50 ratio was significantly less than unity (one sample $t$ test) for all conditions, including regular and sampled stimulation, except for 0 msec delay $(t=0.1,=0.93)$. Unexpectedly, the $\mathrm{P} 30$ ratio, generally expected to be unsuppressed (Kisley et al., 2001), was found significantly less than unity for 0 and 25 msec delays $(t=-6.86, p<$ $0.001 ; t=-3.17, p=0.005)$. The repeated measures (using delays at $0,25,50,75$ msec as a factor) ANOVA on the P30 and P50 peak-to-peak amplitudes for the second tone did not show a significant effect of delay: $\mathrm{P} 30$ ( $\mathrm{df}=3, F=0.86, p=0.46)$ and $\mathrm{P} 50(\mathrm{df}=3, F=0.15, p=0.92)$. Thus the differences in sensory gating ratio were attributable to the effects shown above of phase-triggered stimuli on the first P30 and P50.

\section{Discussion}

We have developed a method that removes the phase artifact present in phaseselective averaging. By selective triggering off of phase, we could increase or decrease P30 and P50 amplitudes selectively and could extract these notoriously small signals with fewer trials than are customarily required. Nevertheless, we remain cautious that the signals we have extracted may differ from the traditionally extracted EPs from nonphaseaveraged methods; further study may reveal novel features in these signals. Not only were we able to affect P30 and P50, but we identified significant effects on gating ratios to paired pulse stimulation as a function of phase.

Although stimuli clearly alter the apparent phase of ongoing EEG after $50 \mathrm{msec}$ (Fig. 2C), the response to stimulation is more than "phase resetting." There are clear neural correlates before 50 msec that are separable from EEG phase by our approach. After $50 \mathrm{msec}$ there is a stimulus-dependent reorganization of EEG that has not yet been fully characterized. We propose a modified schema for evoked potentials, which incorporates our findings with previous results (Fig. 1 AIII).

Nevertheless, our findings strongly suggest that, although these evoked potentials are dependent on ongoing brain activity, phase is not likely to be the only factor that defines the state of the system. Although the phase at threshold was confined within an extremely narrow range, the distribution of phases broadened as delay was increased. We speculate that using several thresholds and delays might improve phase selection accuracy. Yet despite phase dispersion at $50 \mathrm{msec}$ delay, there remained very significant effects on both P30 and P50. These findings demonstrate clearly that, although EEG phase significantly correlates with EP response, consideration of other factors is required to characterize sensory system excitability more fully.

Our results suggest that phase-triggered evoked potential analysis may, in addition to reducing the number of trials required for averaging, produce more robust neural signals and offer a novel
Pooled Phase Histograms

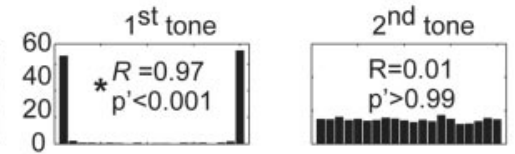

B EP Amplitude, $\mu \mathrm{V}$
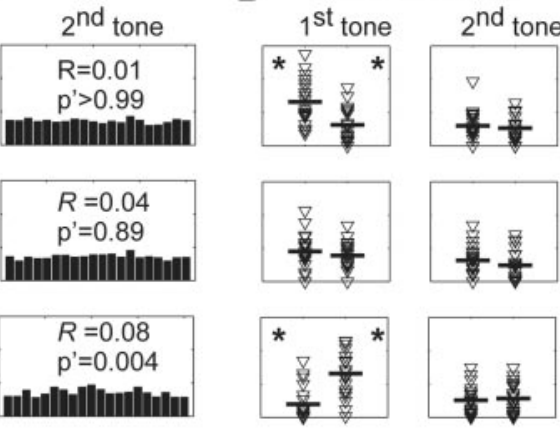

p'=0.00
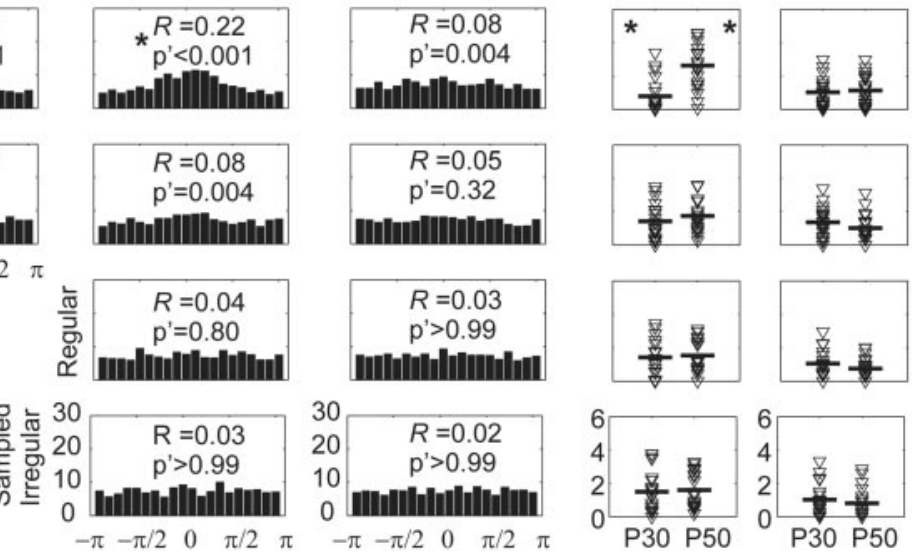

Figure 4. A, Pooled phase histograms from 20 subjects, indicating the distribution (counts) of Hilbert transform-derived phases at the onset of stimuli for 0, 25, 50, and 75 msec delay phase-triggered stimuli as well as regular and sampled irregular

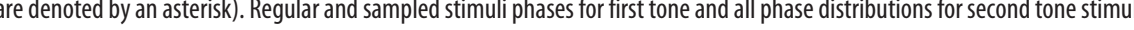
tone stimuli at 0 and 50 msec delays, respectively, and the significant decreases in P30 and P50 amplitudes at 50 and 0 msec delays, respectively, are quantified in the text of the figure.

approach for exploring cognitive physiology. By gating the timing of sensory stimulation to the phase of ongoing EEG, we speculate that phase triggering with unstimulated phase controls introduces a novel means to gate other functional brain imaging modalities such as magnetoencephalogram and functional magnetic resonance imaging as a function of brain state.

\section{References}

Arieli A, Sterkin A, Grinvald A, Aertsen A (1996) Dynamics of ongoing activity: explanation of the large variability in evoked cortical responses. Science 273:1868-1871.

Bechtereva NP, Zontov VV (1962) The relationship between certain forms of potentials and variations in brain excitability (based on EEG, recorded during photic stimuli triggered by rhythmic brain potentials). Electroencephalogr Clin Neurophysiol 14:320-330.

Bendat JS, Piersol AG (2000) Random data: analysis and measurement procedures, Ed 3, pp 518-543. New York: Wiley.

Bishop G (1933) Cyclic changes in excitability of the optic pathway of the rabbit. Am J Physiol 103:213-224.

Brandt ME (1997) Visual and auditory evoked phase resetting of the alpha EEG. Int J Psychophysiol 26:285-298.

Callaway E, Yeager CL (1960) Relationship between reaction time and electroencephalographic alpha phase. Science 132:1765-1766.

Dustman RE, Beck EC (1965) Phase of alpha brain waves, reaction time and visually evoked potentials. Electroencephalogr Clin Neurophysiol 18:433-440.

Fisher NI (1993) Statistical analysis of circular data, p 70. New York: Cambridge UP.

Freedman R, Adler LE, Gerhardt GA, Waldo M, Baker N, Rose GM, Drebing C, Nagamoto H, Bickford-Wimer P, Franks R (1987) Neurobiological studies of sensory gating in schizophrenia. Schizophr Bull 13:669-678. 
Glantz SS, Glantz SA (2001) Primer of biostatistics, p 376. New York: McGraw-Hill.

Jansen BH, Brandt ME (1991) The effect of the phase of prestimulus alpha activity on the averaged visual evoked response. Electroencephalogr Clin Neurophysiol 80:241-250.

Jansen BH, Agarwal G, Hedge A, Boutros NN (2003) Phase synchronization of the ongoing EEG and auditory EP generation. Clin Neurophysiol 114:79-85.

Jerger K, Biggins C, Fein G (1992) P50 suppression is not affected by attentional manipulations. Biol Psychiatry 31:365-377.

Jin Y, Potkin SG, Patterson JV, Sandman CA, Hetrick WP, Bunney WE (1997) Effects of P50 temporal variability on sensory gating in schizophrenia. Psychiatry Res 70:71-81.

Kisley MA, Gerstein GL (1999) Trial-to-trial variability and statedependent modulation of auditory-evoked responses in cortex. J Neurosci 19:10451-10460.

Kisley MA, Olincy A, Freedman R (2001) The effect of state on sensory gating: comparing of waking, REM, and non-REM sleep. Clin Neurophysiol 112:1154-1165.

Lindsley DB (1952) Psychological phenomena and the electroencephalogram. Electroencephalogr Clin Neurophysiol 4:443-456.

Lopes da Silva F (1999) Even-related potentials: methodology and quantification. In: Electroencephalography: basic principles, clinical applications, and related fields (Niedermeyer E, Lopes da Silva F, eds), pp 947-957. Philadelphia: Lippincott, Williams \& Wilkins.

Makeig S (1990) A dramatic increase in the auditory middle latency response at very slow rates. In: Psychophysiological brain research (Brunia
C, Gaillard A, Kok A, eds), pp 56-60. Tilburg, The Netherlands: Tilburg UP.

Makeig S, Westerfield M, Jung TP, Enghoff S, Townsend J, Courchesne E, Sejnowski TJ (2002) Dynamic brain sources of visual evoked responses. Science 295:690-694.

Nagamoto HT, Adler LE, Waldo MC, Griffith J, Freedman R (1991) Gating of auditory response in schizophrenic and normal controls. Schizophr Res 4:31-40.

Netoff TI, Schiff SJ (2002) Decreased neuronal synchronization during experimental seizures. J Neurosci 22:7297-7307.

Polich J (1997) On the relationship between EEG and P300: individual differences, aging, and ultradian rhythms. Int J Psychophysiol 26:299-317.

Rahn E, Başar E (1993) Prestimulus EEG activity strongly influences the auditory evoked vertex response: a new method for selective averaging. Int J Neurosci 69:207-220.

Remond A, Lesèvre N (1967) Variations in average visual evoked potential as a function of the alpha rhythm phase ("autostimulation"). Electroencephalogr Clin Neurophysiol [Suppl] 26:42-52.

Rice DM, Hagstrom EC (1989) Some evidence in support of a relationship between human auditory signal-detection performance and the phase of the alpha cycle. Percept Mot Skills 69:451-457.

Tass PA (1999) Phase resetting in medicine and biology, pp 227-229. Berlin: Springer.

Tsodyks M, Kenet T, Grinvald A, Arieli A (1999) Linking spontaneous activity of single cortical neurons and the underlying functional architecture. Science 286:1943-1946. 